03

\title{
Рассеяние света малыми частицами: эллипсоидальная модель с использованием квазистатического подхода
}

\author{
(C) В.Г. Фарафоонов ${ }^{1}$, В.И. Устимов ${ }^{1}$, М.С. Прокопьева ${ }^{2}$, А.Р. Тулегенов ${ }^{1}$, В.Б. Ильин ${ }^{1,2,3}$ \\ ${ }^{1}$ Государственный университет аэрокосмического приборостроения, \\ 190000 Санкт-Петербург, Россия \\ ${ }^{2}$ Санкт-Петербургский государственный университет, \\ 199034 Санкт-Петербург, Россия \\ ${ }^{3}$ Главная (Пулковская) астрономическая обсерватория РАН, \\ 196140 Санкт-Петербург, Россия \\ e-mail: far@aanet.ru
}

Поступила в редакцию 20.08.2018 г.

Построена эллипсоидальная модель с использованием квазистатического приближения для несферических частиц. Полуоси модельного эллипсоида определяются исходя из требования равенства объемов, а также равенства отношений максимальных продольных и поперечных размеров исходной частицы и модели, что обеспечивает близость их оптических свойств. Данный подход был применен к параллелепипедам, цилиндрам и конусам. Область применимости определялась сравнением результатов численных расчетов по приближенным и строгим методам. В качестве последнего была выбрана аппроксимация дискретными диполями (DDA), которая применима для произвольных несферических частиц. Показано, что область применимости модели для параллелепипедов и цилиндров достаточно широкая для разных параметров задачи. Для конусов она менее пригодна, и от нее следует отказаться для сплюснутых частиц при падении плоской волны ТМ-типа перпендикулярно оси симметрии. В целом предлагаемое приближение дает более точные результаты и имеет большую область применимости при уменьшении относительного показателя преломления и увеличении отношения полуосей „эффективного“ эллипсоида $a_{\mathrm{ef}} / b_{\mathrm{ef}}$, т.е. для сильно вытянутых и сильно сплюснутых прозрачных частиц.

DOI: $10.21883 /$ OS.2018.12.46941.242-18

\section{Введение}

Рассеяние света малыми несферическими частицами представляет большой интерес в различных областях науки $[1,2]$, в особенности в нанооптике [3]. Квазистатический подход (QSA) ранее был использован для вытянутых и сплюснутых сфероидальных частиц [4], а затем для однородных и многослойных эллипсоидов [5]. Он представляет собой обобщение приближений РелеяГанса и Релея, когда поле внутри частицы представляется в виде плоской падающей волны (аппроксимация Релея-Ганса) с учетом поляризуемости частицы в постоянном электрическом поле (аппроксимация Релея). Данный подход предпочтительнее приближения РелеяГанса, так как учитывает поляризуемость рассеивающей частицы и, следовательно, дает правильный статический предел. С другой стороны, он лучше приближения Релея, так как учитывает изменение фазы прямо прошедшего излучения. На первый взляд, область применимости QSA должна быть равна пересечению областей применимости приближений Релея и Релея-Ганса. Однако в действительности это не так, и численный анализ для сфероидов [6] и эллипсоидов [5] показывает, что она, как правило, значительно шире каждой из них. В результате оказалось, что QSA особенно хорошо работает для „мягких“ частиц, сильно отличающихся от шаров. Более того, в случае сильно вытянутых или сплюснутых сфероидов она дает главный член асимптотики рассеянного поля относительно малого параметра $b / a$ - отношения малой полуоси к большой $[9,10]$.

Второй момент связан с построением эллипсоидальной модели для несферической частицы. Здесь, для того чтобы оптические характеристики в дальней зоне для исходной частицы и ее эллипсоидальной модели были бы примерно одинаковыми, требуется учитывать геометрические характеристики. А именно для определения полуосей эллипсоидальной модели $a_{\mathrm{ef}}, b_{\mathrm{ef}}$ и $c_{\mathrm{ef}}$ нужно приравнять друг другу объемы частицы и модели, а также отношения их продольных и поперечных размеров $[9,10]$.

В настоящей работе при построении „эффективной модели для исходной несферической частицы используются оба результата. Сначала из геометрических соображений находится модельный эллипсоид. Затем для этого эллипсоида строится квазистатическое приближение. Далее с помощью численных расчетов на основании строгих методов (разделения переменных [7] и дискретных диполей [11]) и аппроксимации QSA демонстрируется достаточно широкая область применимости последней для частиц разной формы - сфероидов, цилиндров, конусов и параллелепипедов.

\section{1. Основные соотношения}

При построении эллипсоидальной модели исходную частицу заменяем неким „эффективным“ эллипсоидом. 
При этом полуоси $a_{\mathrm{ef}}, b_{\mathrm{ef}}$ и $c_{\text {ef }}$,эффективного“ эллипсоида вычисляются из двух соображений. Во-первых, объемы модельного эллипсоида и несферической частицы должны совпадать:

$$
V_{\mathrm{ef}}=V_{\text {part }}
$$

Во-вторых, для учета формы частицы отношения ее поперечных и продольного размеров должны быть равны отношениям соответствующих эффективных полуосей модели:

$$
\frac{a_{\mathrm{ef}}}{c_{\mathrm{ef}}}=\frac{a_{\mathrm{part}}}{c_{\mathrm{part}}}, \quad \frac{b_{\mathrm{ef}}}{c_{\mathrm{ef}}}=\frac{b_{\mathrm{part}}}{c_{\mathrm{part}}} .
$$

В случае осесимметричной частицы „эффективный“ эллипсоид превращается в вытянутый или сплюснутый „эффективный“ сфероид, при этом получим соотношения

$$
V_{\mathrm{ef}}=V_{\mathrm{part}}, \quad \frac{a_{\mathrm{ef}}}{b_{\mathrm{ef}}}=\frac{a_{\text {part }}}{b_{\text {part }}} .
$$

При определении полуосей $a_{\text {ef }}, b_{\text {ef }}$ и $c_{\text {ef }}$ модельного эллипсоида в случае параллелепипеда со сторонами $a, b, c$ из формул (1), (2) нетрудно получить следующий результат:

$$
a_{\mathrm{ef}}=\sqrt[3]{\frac{3}{4 \pi}} a, \quad b_{\mathrm{ef}}=\sqrt[3]{\frac{3}{4 \pi}} b, \quad c_{\mathrm{ef}}=\sqrt[3]{\frac{3}{4 \pi}} c,
$$

так как здесь имеет место подобное изменение размеров.

Для конечных цилиндров длины $L$ и диаметра $D$ вычисление полуосей „эффективного“ сфероида осуществляется по формулам

$$
a_{\mathrm{ef}}=\sqrt[3]{\frac{3}{16}} D, \quad b_{\mathrm{ef}}=\sqrt[3]{\frac{3}{16}} L
$$

По сравнению с цилиндром конус с высотой $H$ и радиусом $R$ не имеет симметрии относительно плоскости, параллельной $x y$, если его ось симметрии совпадает с осью $z$. „Эффективный“ сфероид строится аналогично случаю цилиндра:

$$
a_{\mathrm{ef}}=\sqrt[3]{\frac{1}{2}} R, \quad b_{\mathrm{ef}}==\sqrt[3]{\frac{1}{16}} H .
$$

Если $H=2 R$, то „эффективный“ сфероид становится шаром. Заметим, что в приведенных выше формулах рассматриваются вытянутые осесимметричные частицы, в противном случае $a_{\text {ef }}$ и $b_{\text {ef }}$ нужно поменять местами.

Ниже используется единый подход к изложению основных аппроксимаций, который состоит в определении характеристик рассеянного излучения по известному полю внутри рассеивателя [12]. При этом внутреннее поле либо аппроксимируется исходя из физических соображений для каждого конкретного случая, либо находится как решение более простой задачи.

Данный подход к построению разных приближений рассеянного поля связан с использованием диадных функций Грина и интегральных уравнений по объему рассеивающего тела:

$$
\mathbf{E}^{(1)}(\mathbf{r})=\int_{V}\left(k_{2}^{2}-k_{1}^{2}\right) \mathbf{E}^{(2)}\left(\mathbf{r}^{\prime}\right) \tilde{G}\left(\mathbf{r}, \mathbf{r}^{\prime}\right) d V^{\prime}
$$

где $k_{1}, k_{2}$ - волновые числа сред вне и внутри частицы, при этом магнитные проницаемости предполагаются равными $\mu=1$, а точка наблюдения $M(\mathbf{r})$ находится вне рассеивающего тела с поверхностью $S$. Диадная функция Грина выражается через функцию Грина скалярного уравнения Гельмгольца

$$
\tilde{G}\left(\mathbf{r}, \mathbf{r}^{\prime}\right)=\left(I+\frac{1}{k_{1}^{2}} \operatorname{grad} \operatorname{div}\right) G\left(\mathbf{r}, \mathbf{r}^{\prime}\right)
$$

где $I$ - единичная матрица. При рассмотрении асимптотического поведения рассеянного поля в дальней зоне $(r \rightarrow \infty)$ вид диадной функции упрощается и уравнение можно записать в виде

$$
\begin{aligned}
\mathbf{E}^{(1)}(\mathbf{r})= & \left(k_{2}^{2}-k_{1}^{2}\right) \frac{\exp \left(i k_{1} r\right)}{4 \pi r}\left(I-\mathbf{i}_{r} \mathbf{i}_{r}^{T}\right) \\
& \times \int_{V} \exp \left(-i k_{1}\left(\mathbf{i}_{r}, \mathbf{r}^{\prime}\right)\right) \mathbf{E}^{(2)}\left(\mathbf{r}^{\prime}\right) d V^{\prime},
\end{aligned}
$$

где $\mathbf{i}_{r}=\mathbf{r} / r-$ вектор столбец, $\mathbf{i}_{r}^{T}-$ вектор-строка, их произведение дает матрицу 3-го порядка.

В рамках квазистатической аппроксимации „исправляется“ приближение Релея-Ганса при помощи учета поляризуемости частицы, т. е. внутреннее поле представляется в виде

$$
\begin{aligned}
\mathbf{E}^{(2)}(\mathbf{r})= & K_{1}\left(\mathbf{E}^{(0)}, \mathbf{i}_{X}\right) \mathbf{i}_{X} \\
& +K_{2}\left(\mathbf{E}^{(0)}, \mathbf{i}_{Y}\right) \mathbf{i}_{Y}+K_{3}\left(\mathbf{E}^{(0)}, \mathbf{i}_{Z}\right) \mathbf{i}_{Z},
\end{aligned}
$$

где $\left(\mathbf{i}_{\mathrm{X}}, \mathbf{i}_{\mathrm{Y}}, \mathbf{i}_{\mathrm{Z}}\right)$ - орты декартовой системы $(X, Y, Z)$, оси которой направлены вдоль главных осей эллипсоида. Коэффициенты $K_{j}$ связаны с соответствующими геометрическими факторами в приближении Релея:

$$
K_{j}=\left(1+L_{j}(\varepsilon-1)\right)^{-1}, j=1,2,3,
$$

где $\varepsilon=\varepsilon_{2} / \varepsilon_{1}-$ относительная диэлектрическая проницаемость вещества частицы (считаем, что магнитная проницаемость $\mu=1)$. Геометрические факторы сфероидов и эллипсоидов хорошо известны [1].

Рассеянное поле в дальней зоне в квазистатическом приближении вычисляется по формуле

$$
\begin{gathered}
\mathbf{E}^{(1)}(\mathbf{r})=\left(k_{2}^{2}-k_{1}^{2}\right) \frac{\exp \left(i k_{1} r\right)}{4 \pi r} \\
\times V\left(I-\mathbf{i}_{r} \mathbf{i}_{r}^{T}\right) \mathbf{E}^{(2)}(\mathbf{r}) G(\vartheta, \phi),
\end{gathered}
$$


где введена функция, называемая форм-фактором,

$$
G(\vartheta, \phi)=\frac{1}{V} \int_{V} \exp \left(i k_{1}\left(\mathbf{i}_{k_{1}}-\mathbf{i}_{r}, \mathbf{r}^{\prime}\right)\right) d V^{\prime},
$$

$\mathbf{i}_{k_{1}}=\mathbf{k}_{1} / k_{1}, \quad(r, \vartheta, \phi)-$ сферическая система координат, связанная с падающим излучением, т. е. ось $z$ совпадает с направлением распространения плоской волны.

Для эллипсоидальных частиц формфактор вычисляется в явном виде:

$$
G(\vartheta, \phi)=G(u)=\frac{3}{u^{3}}(\sin u-u \cos u),
$$

где

$$
u=2 k_{1} \sin \frac{\vartheta}{2} \sqrt{a^{2}\left(\mathbf{i}_{\mathrm{X}}, \mathbf{i}_{\mathrm{b}}\right)^{2}+b^{2}\left(\mathbf{i}_{\mathrm{Y}}, \mathbf{i}_{\mathrm{b}}\right)^{2}+c^{2}\left(\mathbf{i}_{\mathrm{Z}}, \mathbf{i}_{\mathrm{b}}\right)^{2}},
$$

$\mathrm{a}\left(\mathbf{i}_{\mathrm{X}}, \mathbf{i}_{\mathrm{b}}\right),\left(\mathbf{i}_{\mathrm{Y}}, \mathbf{i}_{\mathrm{b}}\right),\left(\mathbf{i}_{\mathrm{Z}}, \mathbf{i}_{\mathrm{b}}\right)$ - косинусы углов между соответствующими осями эллипсоида и биссектрисой дополнительного угла рассеяния (т.е. угла между направлением рассеянного излучения и направлением, противоположенным направлению распространения падающего излучения).

Сечение рассеяния определяется интегрированием интенсивности рассеянного излучения по бесконечно удаленной сфере:

$$
C_{\mathrm{sca}}=\int_{\Omega} \int\left|\mathbf{E}^{(1)}(\mathbf{r})\right|^{2} d \omega
$$

Для вытянутого сфероида с полуосями $a$ и $b$ в случае ТЕ-моды получим

$$
\begin{aligned}
& Q_{\text {sca }, \mathrm{TE}}=\frac{C_{\mathrm{sca}, \mathrm{TE}}}{\pi r_{v}^{2}}=\frac{x_{v}^{4}}{9 \pi}\left|\frac{(\varepsilon-1)}{1+(\varepsilon-1) L_{2}}\right|^{2} \\
& \times \int_{0}^{2 \pi} \int_{0}^{\pi}\left(\cos ^{2} \varphi+\cos ^{2} \theta \sin ^{2} \varphi\right) G^{2}(u) \sin \theta d \theta d \varphi .
\end{aligned}
$$

В формуле (16) аргумент функции $G(u)$ определяется выражениями (14), которые в системе координат $(r, \theta, \varphi)$, связанной с вытянутым сфероидом, имеет вид

$$
u=(a / b)^{2 / 3} x_{v} \sqrt{\begin{array}{r}
(\cos \theta-\cos \alpha)^{2}+(b / a)^{2}\left(\sin ^{2} \theta+\right. \\
\left.+\sin ^{2} \alpha-2 \sin \theta \sin \alpha \cos \varphi\right)
\end{array}} .
$$

Здесь использовался единый подход к определению размера разных по форме частиц с помощью дифракционного параметра:

$$
x_{v}=k_{1} r_{v}=\frac{2 \pi r_{v}}{\lambda}
$$

где $r_{v}$ - радиус эквиобъемного шара (т.е. $4 \pi / 3 r_{v}^{3}=$ $\left.=V_{\text {part }}=4 \pi / 3 a b^{2}\right), \lambda$ - длина волны излучения.
Для ТМ-моды нормированное сечение рассеяния находится аналогично:

$$
\begin{aligned}
& Q_{\text {sca }, \mathrm{TM}}=\frac{C_{\text {sca }, \mathrm{TM}}}{\pi r_{v}^{2}}=\frac{x_{v}^{4}}{9 \pi}\left\{\left|\frac{(\varepsilon-1)}{1+(\varepsilon-1) L_{1}}\right|^{2} \cos ^{2} \alpha\right. \\
& \times \int_{0}^{2 \pi} \int_{0}^{\pi}\left(\sin ^{2} \varphi+\cos ^{2} \theta \cos ^{2} \varphi\right) G^{2}(u) \sin \theta d \theta d \varphi \\
& \times 2 \operatorname{Re}\left(\frac{(\varepsilon-1)}{1+(\varepsilon-1) L_{1}} \frac{(\varepsilon-1)}{1+(\varepsilon-1) L_{3}}\right) \sin \alpha \cos \alpha \\
& \times \int_{0}^{2 \pi} \int_{0}^{\pi} G^{2}(u) \cos \theta \sin ^{2} \theta \cos \varphi d \theta d \varphi \\
& \left.\times \sin ^{2} \alpha\left|\frac{(\varepsilon-1)}{1+(\varepsilon-1) L_{3}}\right|^{2} \int_{0}^{2 \pi} \int_{0}^{\pi} G^{2}(u) \sin ^{3} \theta d \theta d \varphi\right\} .
\end{aligned}
$$

Для сплюснутых сфероидов полуоси $a$ и $b$ нужно поменять местами.

В рамках приближения Релея аргумент формфактора $u=0$, а сама функция $G(0)=1$. В результате выражения (16) и (19) совпадают с аналогичными факторами эффективности в релеевском приближении. Поскольку $G^{2}(u) \leq 1$, то факторы рассеяния в квазистатическом приближении всегда меньше, чем в статическом $Q_{\mathrm{sca}}^{\mathrm{QSA}} \leq Q_{\mathrm{sca}}^{\mathrm{R}}$

Сечения поглощения определяются интегрированием поля излучения внутри частицы по объему частицы:

$$
C_{\mathrm{abs}}=k_{1} \int_{\mathrm{V}} \operatorname{Im}(\varepsilon-1)\left|\mathbf{E}^{(2)}(\mathbf{r})\right|^{2} d V
$$

Поскольку модули напряженности электрического поля в релеевском и квазистатическом приближениях совпадают, то в обоих случаях получим одинаковый результат.

В случае ТЕ-моды зависимость факторов рассеяния от ориентации частицы (угла падения $\alpha$ ) связана только с аргументом $и$ функции $G(u)$ (см. (16) и (17)), а для малых частиц, т.е. в приближении Релея, она отсутствует.

При падении плоской волны вдоль оси вращения $(\alpha=0)$ различия между поляризациями исчезают, при этом интеграл по азимутальному углу можно вычислить непосредственно:

$$
Q_{\text {sca }}=\frac{x_{v}^{4}}{9}\left|\frac{(\varepsilon-1)}{1+(\varepsilon-1) L_{1}}\right|^{2} \int_{0}^{\pi}\left(1+\cos ^{2} \theta\right) G^{2}(u) \sin \theta d \theta,
$$

где

$$
u=(a / b)^{2 / 3} x_{v} \sqrt{(\cos \theta-1)^{2}+(b / a)^{2} \sin ^{2} \theta}
$$


Если плоская волна падает перпендикулярно оси вращения $(\alpha=90)$, то получим

$Q_{\text {sca, } \mathrm{TM}}=\frac{x_{v}^{4}}{9 \pi}\left\{\left|\frac{(\varepsilon-1)}{1+(\varepsilon-1) L_{3}}\right|^{2} \int_{0}^{2 \pi} \int_{0}^{\pi} G^{2}(u) \sin ^{3} \theta d \theta d \varphi\right\}$,

где

$u=(a / b)^{2 / 3} x_{v} \sqrt{\cos ^{2} \theta+(b / a)^{2}\left(\sin ^{2} \theta+1-2 \sin \theta \cos \varphi\right)}$.

Для сильно вытянутых частиц $(b / a \ll 1)$ геометрические факторы нетрудно найти:

$$
L_{1}=L_{2}=1 / 2, L_{3}=0,
$$

при этом аргумент $u \approx x_{a}|\cos \alpha-\cos \theta|$. При параллельной и перпендикулярной ориентациях частицы получим $u \approx x_{a}(1-\cos \theta)$ и $u \approx x_{a}|\cos \theta|$ соответственно. Модуль можно убрать, так как функция $G^{2}(u)$ четна. Здесь фигурирует новый дифракционный параметр, связанный с большим размером частицы,

$$
x_{a}=k_{1} a=\frac{2 \pi a}{\lambda} .
$$

Для сильно сплюснутых частиц $(b / a \ll 1)$ геометрические факторы $L_{1}=L_{2}=0, L_{3}=1$, при этом $u \approx x_{a} \sqrt{\sin ^{2} \theta+1-2 \sin \theta \cos \varphi}$. В меридиальной плоскости $(\varphi=0$ и $\varphi=\pi)$ последняя формула принимает вид $u \approx x_{a} \sin \theta$ и $u \approx x_{a}(1-\sin \theta)$ соответственно при параллельной и перпендикулярной ориентациях частицы. Заметим, что для сильно вытянутых и сплюснутых частиц значения аргумента $u$ совпадают для прямо прошедшего и перпендикулярного направлений рассеянного излучения.

\section{2. Численные результаты и обсуждение}

Численные расчеты проводились с использованием как квазистатического приближения QSA, так и строгого метода DDA [11], который позволяет моделировать рассеяние света частицами произвольной формы, а также имеет упрощенную процедуру задания осесимметричных частиц. При этом в большинстве случаев конечными результатами расчетов были факторы рассеяния $Q_{\text {sca }}$.

Кроме того, для сфероидов использовался метод SVM [7], с помощью которого характеристики рассеянного излучения можно вычислять с высокой точностью в широкой области изменения параметров частицы. С его помощью было проведено детальное исследование областей применимости QSA и релеевского приближения [6]. Оказалось, что при небольших отношениях полуосей сфероида $a / b$ последнее может иметь более широкую область применимости, но уже при $a / b \geq 3$ аппроксимация QSA имеет преимущество при относительной

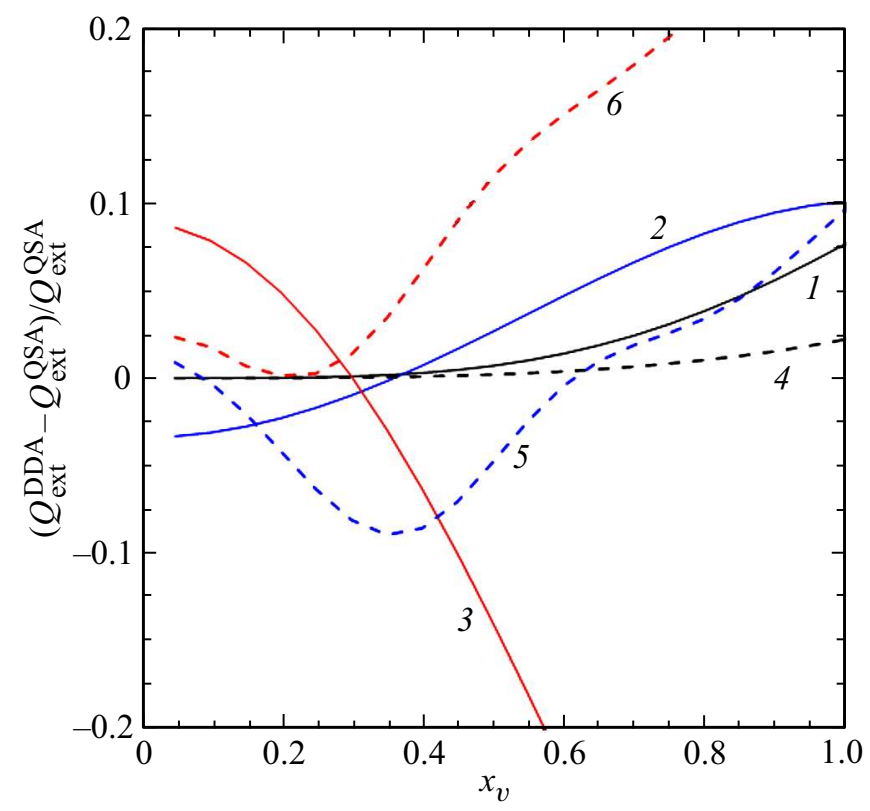

Рис. 1. Относительная погрешность $\delta$ расчета факторов эффективности рассеяния несферических частиц с показателем преломления $m=1.3$ и углом падения излучения $\alpha=0$ в зависимости от дифракционного параметра $x_{v}$. В случае кривых 1,4 : параллелепипеды с отношениями $a / b=0.1$ (一) и $a / b=10(--)$; для кривых 2,5: цилиндры с отношениями $L / D=0.1(-)$ и $L / D=10(--)$ и для кривых 3,6 : конусы с отношениями $H / D=0.1(-)$ и $H / D=10(--)$.

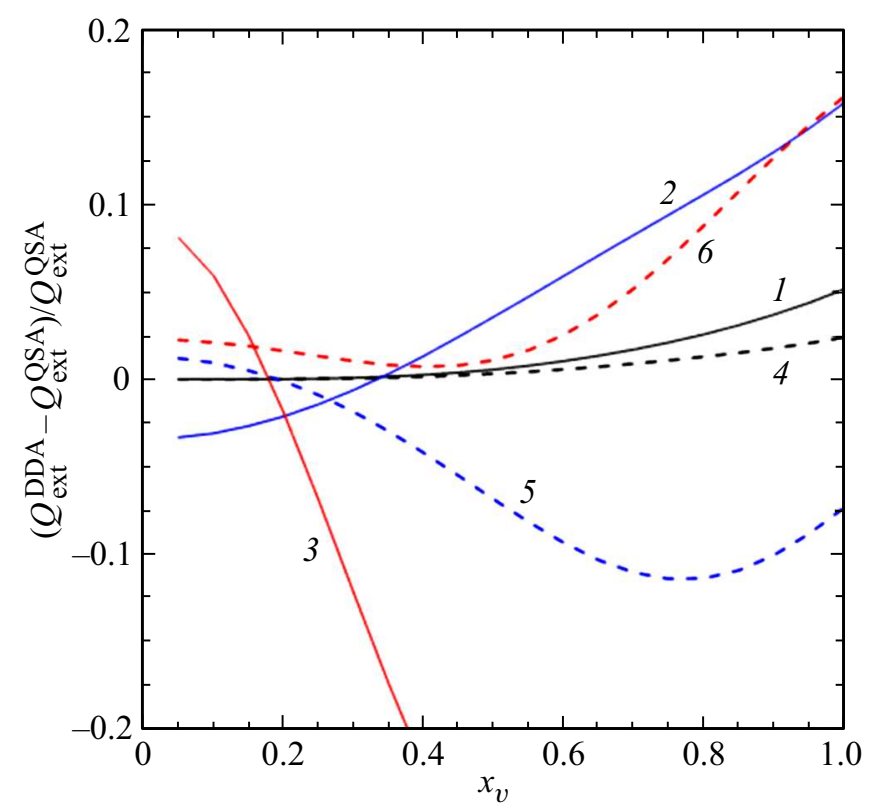

Рис. 2. То же, что на рис. 1 , но для угла падения $\alpha=90^{\circ}$ и падающей волны ТЕ-типа.

ошибке $\left|Q_{\mathrm{sca}}^{\mathrm{approx}} / Q_{\mathrm{sca}}^{\mathrm{ext}}-1\right| \geq 0.01$. При увеличении относительной ошибки до $10 \%$ область применимости QSA значительно увеличивается. В целом, наилучшие результаты QSA дает с увеличением степени асферичности 


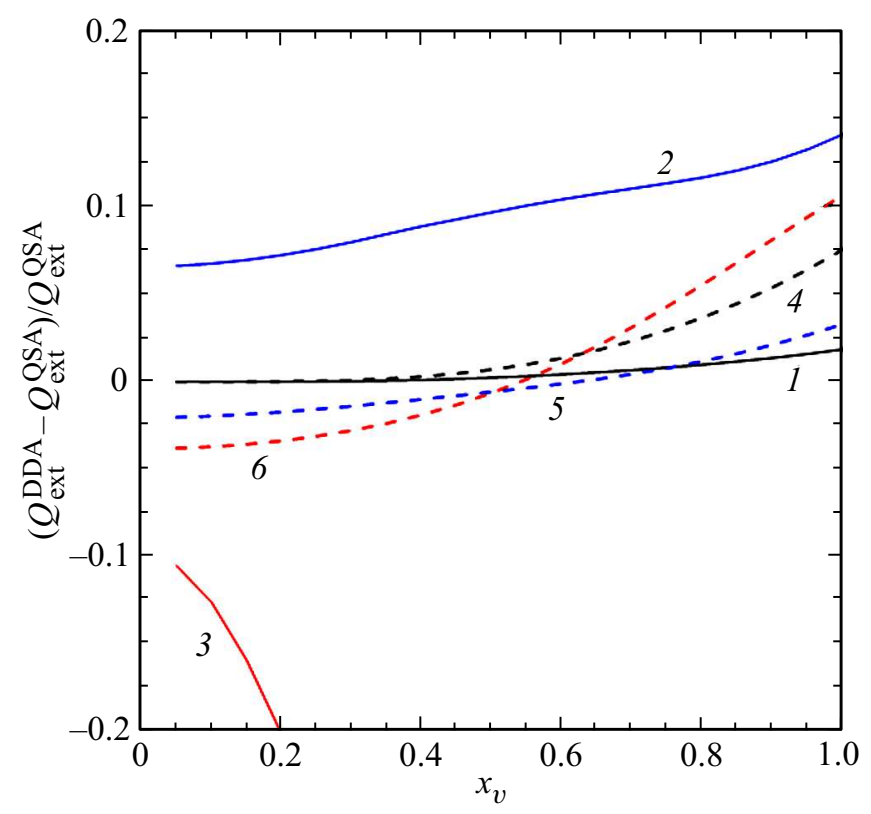

Рис. 3. То же, что на рис. 1 , но для угла падения $\alpha=90^{\circ}$ и падающей волны ТМ типа.

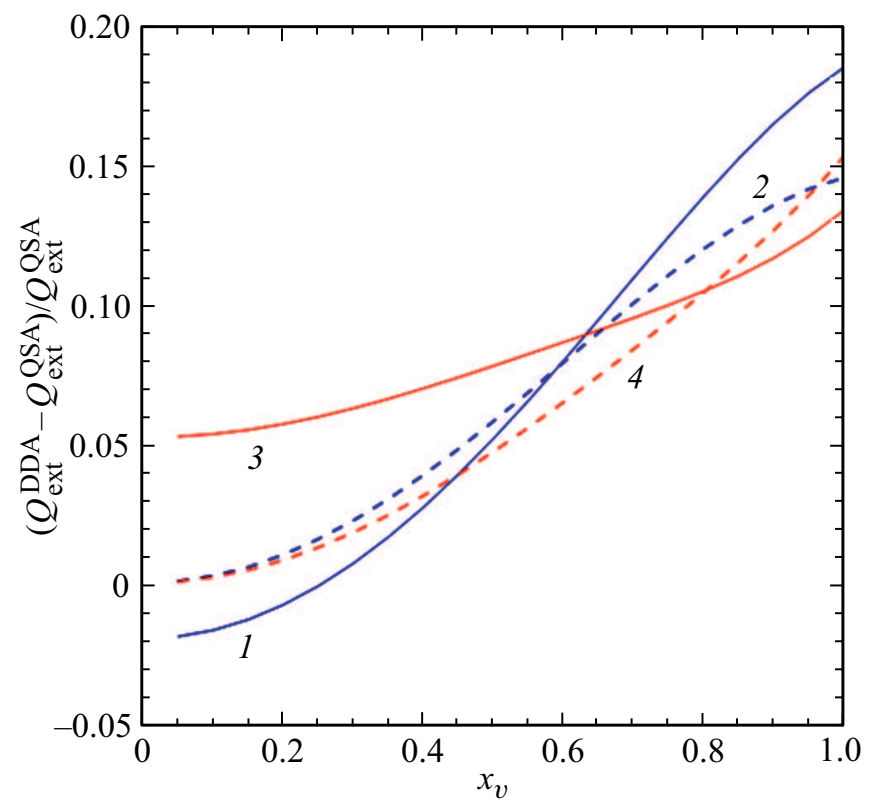

Pис. 4. То же, что на рис. 1 , но для параллелепипедов с тремя разными гранями $-a: b: c=1: 2: 3$. В случае кривых 1,2 : $\alpha=0$, для кривых 3,4: $\alpha=90$, Волны ТМ (-) и ТЕ (- - ) типов.

$a / b$ и уменьшением показателя преломления $m$. Ранее в работе [13] рассматривалось рассеяние света малыми цилиндров в рамках приближения Релея. Ниже основное внимание уделяется квазистатическому приближению, которое можно применять в более широкой области.

Расчеты проводились для частиц разной формы - цилиндров, конусов и параллелепипедов. В нашей моделе разные несферические частицы, имеющие одинаковый объем (1) и отношение поперечных и продольного размеров (2), соответствуют одному „эффективному“ эллипсоиду. В силу этого у них должны быть примерно одинаковые характеристики рассеяния в дальней зоне, в нашем случае - факторы эффективности рассеяния $Q_{\mathrm{sca}}^{\mathrm{DDA}}$, которые должны незначительно отличаться от соответствующих факторов $Q_{\mathrm{sca}}^{\mathrm{QSA}}$, определяемых в рамках предлагаемой модели. Относительная погрешность будет рассчитываться по следующей формуле:

$$
\delta=\left|Q_{\mathrm{sca}}^{\mathrm{DDA}} / Q_{\mathrm{sca}}^{\mathrm{QSA}}-1\right|,
$$

где методом DDA вычисляются факторы разных несферических частиц, а аппроксимация QSA используется для модельного эллипсоида или сфероида.

Для достаточно сплюснутых и вытянутых параллелепипедов с отношением сторон $a_{\mathrm{ef}} / b_{\mathrm{ef}}=10$ эллипсоидальная модель с использованием квазистатического приближения дает отличные результаты. Относительная погрешность для частиц с дифракционными параметрами $x_{v} \leq 1.0$ составляет меньше 0.08 , т.е. $8 \%$, при разных углах падения и поляризации падающего излучения (рис. 1-3). Для параллелепипедов с тремя разными гранями и сравнительно небольшими отношениями сторон соответствующая погрешность достигает $10 \%$ при дифракционных параметрах $x_{v} \leq 0.6$ (рис. 4). Аналогичная картина имеет место для симметричных параллелепипедов с такими же отношениями сторон (рис. 5).

В случае цилиндрических частиц ситуация с применением QSA примерно такая же, как и для симметричных параллелепипедов, но с тенденцией ухудшения. Так для сильно сплюснутых и вытянутых цилиндров относительная погрешность не превышает 10\% при дифракционных параметрах $x_{v} \leq 0.6$, а дальше, при $x_{v}=0.6-1.0$,

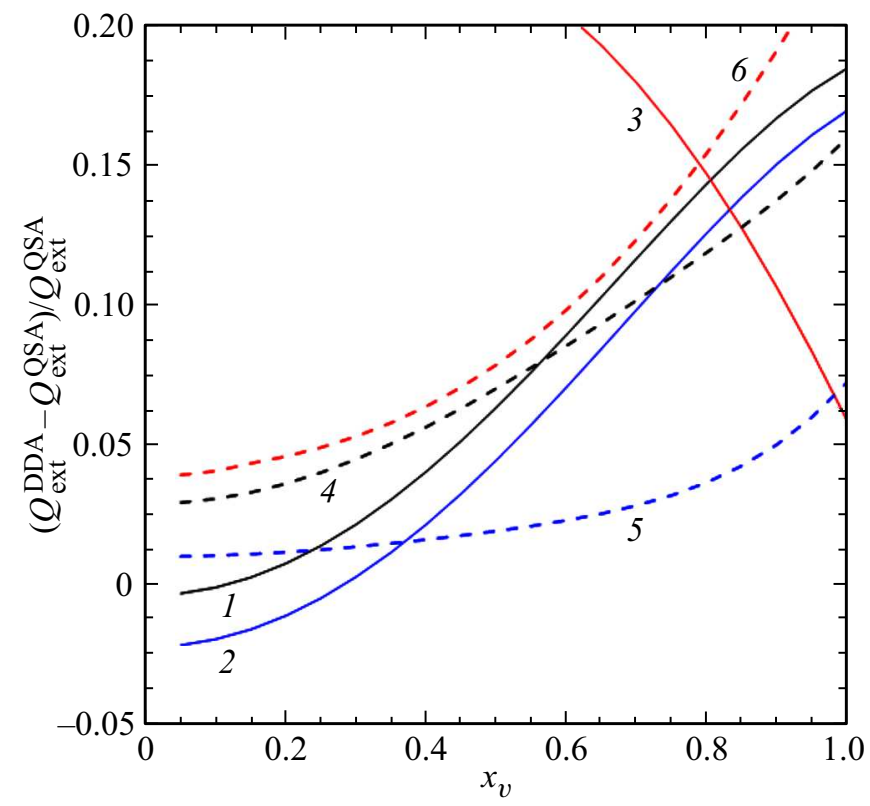

Рис. 5. То же, что на рис. 1 , но для частиц с отношениями $a / b=0.5(-)$ и $a / b=2(--)$. 
она может достигать $15 \%$ (рис. 1-3). Если отношения размеров невелики, например $L / D=2.0$, то область применимости QSA сужается примерно также, как для параллелепипедов (рис. 5). Что касается конусов, то она менее пригодна, при этом от нее следует отказаться для сплюснутых частиц при падении плоской волны ТМ-типа перпендикулярно оси симметрии (рис. 1-3,5).

Заметим, что рассматриваемые частицы могут иметь достаточно большие продольные размеры по сравнению с длиной волны падающего излучения. Объемный дифракционный параметр $x_{v}$ и соответствующий линейный параметр $x_{a}$ связаны соотношением: $x_{a} / x_{v}=\left(a_{\mathrm{ef}} / b_{\mathrm{ef}}\right)^{1 / 3}$ для сплюснутых частиц и $x_{a} / x_{v}=\left(a_{\mathrm{ef}} / b_{\mathrm{ef}}\right)^{2 / 3}$ для вытянутых. Так для сплюснутых частиц с отношением $a_{\text {ef }} / b_{\text {ef }}=10$ и объемным дифракционным параметром $x_{v}=1.0$ получим линейный параметр $x_{a} \approx 2.15$, а для аналогичных вытянутых частиц уже $x_{a} \approx 4.65$. Такие длинные частицы не могут рассматриваться в приближении Релея.

На основании выполненного аналитического и численного анализа эллипсоидальной модели с использованием квазистатической аппроксимации можно сделать следующие выводы.

1. Проведенное сравнение результатов численных расчетов по строгим и приближенным алгоритмам для несферических частиц показало, что в рамках эллипсоидальной модели вычисления в соответствии с квазистатическим приближением имеют достаточно малую относительную погрешность в широкой области изменения параметров задачи и, как правило, предлагаемая модель имеет преимущество перед приближением Релея. Это утверждение справедливо для параллелепипедов и цилиндров. В то же время для конусов данный подход имеет ограниченную область применимости.

2. Квазистатическое приближение дает более точные результаты для прозрачных частиц, т.е. с уменьшением разности показателей преломления между средами, и для сильно вытянутых и сильно сплюснутых частиц, т.е. сильно отличающихся от шаров.

3. Область применимости эллипсоидальной модели для малых осесимметричных частиц значительно шиpe, чем таковая для строгих методов ЕBCM и SVM, использующих сферический базис для представления полей $[9,10]$.

Работа была поддержана в 2018 г. грантом ГУАП и грантами РФФИ 16-02-00194 и 18-52-52006. Авторы благодарны М. Юркину за разрешение использовать программу ADDA.

\section{Список литературы}

[1] Борен К., Хафбфмен Д. Поглощение и рассеяние света малыми частицами. М.: Мир, 1986.

[2] Mishchenko M.I., Travis L.D., Lacis A.A. Scattering, Absorption and Emission of Light by Small Particles. Cambridge: Cambridge Univ. Press., 2002.
[3] Климов В.В. Наноплазмоника. М.: Физматлит, 2009.

[4] Фарафбонов В.Г. // Опт. и спектр. 1994. Т. 77. С. 455.

[5] Фарафонов В.Г., Ильин В.Б., Прокопьева М.С. // Опт. и спектр. 2002. Т. 92. № 3. С. 621.

[6] Вощинников Н.В., Фарафбонов В.Г. // Опт. и спектр. 2000. T. 88. № 1. C. 78.

[7] Voshchinnikov N.V., Farafonov V.G. // Astrophys. Space Sci., 1993. V. 204. P. 19.

[8] Farafonov V.G. Application of non-orthogonal bases in the theory of light scattering by spheroidal particles. In Kokhanovsky A. (Ed.), Light Scattering Reviews 8, London: Springer-Praxis, p. 189-266. 2013.

[9] Фарафонов В.Г., Ильин В.Б., Устимов В.И., Тулегенов А.Р. // Опт. и спектр. 2017. Т. 122. № 4. С. 506.

[10] Фарафбонов В.Г., Ильин В.Б., Устимов В.И., Соколовская М.В. // Опт. и спектр. 2018. Т. 124. С.

[11] Yurkin M.A., Hoekstra A.G. // JQSRT. 2011. V. 112. P. 2234.

[12] Исимару A. Распространение и рассеяние волн в случайно-неоднородной среде. Т. 1. М.: Мир, 1981.

[13] Sihvola A., Venermo J., Ylä-Oijala P. // Microwav. Tech. Lett. 2004. V. 41. P. 245. 